\title{
PSEUDOCOMPACT AND COUNTABLY COMPACT ABELIAN GROUPS: CARTESIAN PRODUCTS AND MINIMALITY
}

\author{
DIKRAN N. DIKRANJAN AND DMITRII B. SHAKHMATOV
}

\begin{abstract}
Denote by $\mathscr{G}$ the class of all Abelian Hausdorff topological groups. A group $G \in \mathscr{G}$ is minimal (totally minimal) if every continuous group isomorphism (homomorphism) $i: G \rightarrow H$ of $G$ onto $H \in \mathscr{G}$ is open. For $G \in \mathscr{G}$ let $\kappa(G)$ be the smallest cardinal $\tau \geq 1$ such that the minimality of $G^{\tau}$ implies the minimality of all powers of $G$. For $\mathscr{Q} \subset \mathscr{G}, \mathscr{Q} \neq \varnothing$, we set $\kappa(\mathscr{Q})=\sup \{\kappa(G): G \in \mathscr{G}\}$ and denote by $\alpha(\mathscr{Q})$ the smallest cardinal $\tau \geq 1$ having the following property: If $\left\{G_{i}: i \in I\right\} \subset \mathscr{Q}, I \neq \varnothing$, and each subproduct $\Pi\left\{G_{i}: i \in J\right\}$, with $J \subset I, J \neq \varnothing$, and $|J| \leq \tau$, is minimal, then the whole product $\prod\left\{G_{i}: i \in I\right\}$ is minimal. These definitions are correct, and $\kappa(G) \leq 2^{\omega}$ and $\kappa(\mathscr{Q}) \leq \alpha(\mathscr{Q}) \leq 2^{\omega}$ for all $G \in \mathscr{G}$ and any $\mathscr{Q} \subset \mathscr{G}, \mathscr{Q} \neq \varnothing$, while it can happen that $\kappa(\mathscr{Q})<\alpha(\mathscr{Q})$ for some $\mathscr{Q} \subset \mathscr{G}$. Let $\mathscr{C}=\{G \in \mathscr{G}: G$ is countably compact $\}$ and $\mathscr{P}=\{G \in \mathscr{G}: G$ is pseudocompact $\}$. If $G \in \mathscr{C}$ is minimal, then $G \times H$ is minimal for each minimal (not necessarily Abelian) group $H$; in particular, $G^{n}$ is minimal for every natural number $n$. We show that $\alpha(\mathscr{C})=\omega$, and so either $\kappa(\mathscr{C})=1$ or $\kappa(\mathscr{C})=\omega$. Under Lusin's Hypothesis $2^{\omega_{1}}=2^{\omega}$ we construct $\left\{G_{n}: n \in \mathbb{N}\right\} \subset \mathscr{P}$ and $H \in \mathscr{P}$ such that: (i) whenever $n \in \mathbb{N}, G_{n}^{n}$ is totally minimal, but $G_{n}^{n+1}$ is not even minimal, so $\kappa\left(G_{n}\right)=n+1$; and (ii) $H^{n}$ is totally minimal for each natural number $n$, but $H^{\omega}$ is not even minimal, so $\kappa(H)=\omega$. Under $\mathrm{MA}+\neg \mathrm{CH}$, conjunction of Martin's Axiom with the negation of the Continuum Hypothesis, we construct $G \in \mathscr{P}$ such that $G^{\tau}$ is totally minimal for each $\tau<2^{\omega}$, while $G^{2^{\omega}}$ is not minimal, so $\kappa(G)=2^{\omega}$. This yields $\alpha(\mathscr{P})=\kappa(\mathscr{P})=2^{\omega}$ under MA $+\neg \mathrm{CH}$. We also present an example of a noncompact minimal group $G \in \mathscr{C}$, which should be compared with the following result obtained by the authors quite recently: Totally minimal groups $G \in \mathscr{C}$ are compact.
\end{abstract}

\section{INTRODUCTION}

All topological groups considered in this paper are assumed to be Hausdorff. A topological group $G$ is said to be minimal (totally minimal) if every continuous group isomorphism (homomorphism) $i: G \rightarrow H$ onto a topological group $H$ is open [S1, DP]. Clearly every compact group is totally minimal and every totally minimal group is minimal. In the converse direction, Prodanov and Stoyanov [PS1] showed that minimal Abelian groups are precompact.

Received by the editors October 12, 1989 and, in revised form, November 13, 1990.

1980 Mathematics Subject Classification (1985 Revision). Primary 54B10, 54D25, 54D30; Secondary $22 \mathrm{~A} 05,22 \mathrm{C} 05,54 \mathrm{~A} 35$.

Key words and phrases. Countably compact space, pseudocompact space, $\omega$-bounded space, topological group, minimal group, totally minimal group, Cartesian product, Tychonoff product, cardinal invariant. 
A difficult problem in the theory of minimal topological groups, set by Choquet, is to determine when minimality is preserved by Cartesian products. Doitchinov [Do] showed that the product of two minimal (Abelian) groups need not be minimal. Stoyanov [S3] introduced the notion of a perfectly minimal group, this is a minimal group $G$ such that for every minimal group $H$ the product $G \times H$ is minimal. It was proved earlier by Stephenson [S2] that every minimal group which is a union of its compact subgroups is perfectly minimal (see Theorem 1.2 for a generalization of this result).

Clearly all finite powers of a perfectly minimal group are minimal. It was shown in [S3] that this cannot be extended to infinite powers. In the same paper Stoyanov established that all powers of an Abelian group $G$ are minimal provided that $G^{\mathfrak{c}}$ is minimal (here and in the sequel $\mathfrak{c}$ stays for denoting the cardinality of the continuum). Dikranjan improved his result as follows: Whenever $\left\{G_{i}: i \in I\right\}$ is a nonempty family of topological Abelian groups, the product $\prod\left\{G_{i}: i \in I\right\}$ is minimal provided that each subproduct $\prod\left\{G_{i}: i \in J\right\}$, with $J \subseteq I, J \neq \varnothing$, and $|J| \leq \mathfrak{c}$, is minimal (announced in [D]; see also [DS1, Theorem 3.7] or [DPS, Theorem 6.2.6] for a proof). This justifies the following

0.1. Main definition. (i) For a topological Abelian group $G$ let $\kappa(G)$ be the smallest cardinal $\tau \geq 1$ such that the minimality of $G^{\tau}$ implies the minimality of all powers of $G$.

(ii) For a nonempty class $\mathscr{Q}$ of topological Abelian groups define $\kappa(\mathscr{Q})=$ $\sup \{\kappa(G): G \in \mathscr{Q}\}$.

(iii) For a nonempty class $\mathscr{Q}$ of topological Abelian groups denote by $\alpha(\mathscr{Q})$ the smallest cardinal $\tau \geq 1$ satisfying the following condition: Whenever $\left\{G_{i}: i \in I\right\} \subset \mathscr{Q}, I \neq \varnothing$, and each subproduct $\prod\left\{G_{i}: i \in J\right\}$, with $J \subset I$, $J \neq \varnothing$, and $|J| \leq \tau$, is minimal, then the whole product $\prod\left\{G_{i}: i \in I\right\}$ is also minimal.

(iv) Let $\alpha=\alpha(\mathscr{G})$ and $\kappa=\kappa(\mathscr{G})$, where $\mathscr{G}$ is the class of all topological Abelian groups.

Observe that if all powers $G^{\tau}$ of a topological Abelian group $G$ are minimal, then $\kappa(G)=1$; otherwise $\kappa(G)$ coincides with the smallest cardinal $\tau$ for which $G^{\tau}$ fails to be minimal. This is the reason why $\kappa(G)$ was called the critical power of minimality of $G$ in [DS1] (see also [DPS, Chapter 6.3]). Cardinals $\kappa(G), \kappa(\mathscr{Q})$, and $\kappa$ were introduced in [DS1], cardinal $\alpha$ was considered (although in somewhat different form) in [D], and was defined for the first time, jointly with $\alpha(\mathscr{Q})$, in [DPS, Exercise 6.5.31]. The next proposition clarifies interrelations between the cardinals defined above.

0.2. Proposition. (i) $\alpha \leq \mathfrak{c}$ and $\kappa \leq \mathfrak{c}$.

(ii) If $\mathscr{Q}$ and $\mathscr{R}$ are nonempty classes of topological Abelian groups and $\mathscr{Q} \subseteq \mathscr{R}$, then $\alpha(\mathscr{Q}) \leq \alpha(\mathscr{R})$ and $\kappa(\mathscr{Q}) \leq \kappa(\mathscr{R}) ;$ in particular, $\alpha(\mathscr{Q}) \leq \alpha$ and $\kappa(\mathscr{Q}) \leq \kappa$.

(iii) If $\mathscr{Q}$ is a class of topological Abelian groups and $G \in \mathscr{Q}$, then $\kappa(G) \leq$ $\kappa(\mathscr{Q})$; in particular, $\kappa(G) \leq \kappa$.

(iv) $\kappa(\mathscr{Q}) \leq \alpha(\mathscr{Q})$ for every nonempty class $\mathscr{Q}$ of topological Abelian groups; in particular, $\kappa \leq \alpha$.

Proof. (i) follows from results of Dikranjan and Stoyanov cited above, and (ii)-(iv) immediately follow from Main Definition 0.1 . 
Since the definitions of $\alpha(\mathscr{Q})$ and $\kappa(\mathscr{Q})$ are similar to each other, the natural question arises of whether the inequality in Proposition 0.2(iv) can be strengthened to the equality. Theorem 1.11 answers this question in the negative, thus justifying the introduction of both cardinals into our considerations.

The problem to exactly determine the cardinal $\kappa$ was posed (without using $\kappa$ ) by Prodanov and Stoyanov [PS2]. It was shown in [DS1] that $\kappa=$ $\kappa(\{$ separable metrizable Abelian groups $\})$ and moreover, for every cardinal $\tau$ satisfying $2^{\tau}=\mathfrak{c}$ a separable metrizable minimal group $G$ with $\kappa(G)>\tau$ was constructed; in particular, this yields that $\kappa>\omega$. In the present paper we study the cardinals $\alpha(\mathscr{C}), \alpha(\mathscr{P}), \kappa(\mathscr{C})$, and $\kappa(\mathscr{P})$, where $\mathscr{C}$ and $\mathscr{P}$ denote the classes of countably compact Abelian groups and pseudocompact Abelian groups, respectively. (Recall that a topological space $X$ is countably compact $[\mathrm{F}]$ iff any countable open cover of $X$ admits a finite subcover, and it is pseudocompact $[\mathrm{H}]$ iff every real-valued continuous function defined on $X$ is bounded; compact spaces are countably compact and countably compact spaces are pseudocompact-these facts together with supplementary information about countably compact and pseudocompact spaces, including, in particular, examples which show that neither of the above implications is reversible, can be found in [E, Chapter 3.10].)

It requires some effort to construct a noncompact, countably compact (or even pseudocompact), minimal topological group. Examples of noncommutative, countably compact, noncompact, minimal groups were given by Comfort and Grant [GG] and Guran [G]. The first example of a pseudocompact, noncompact, totally minimal Abelian group was constructed by Comfort and Soundararajan [CS] (see also [CRb]), and examples of countably compact, noncompact, minimal Abelian groups seem to be unknown (see Corollary 1.6 for such an example). It should also be noted that countably compact, totally minimal Abelian groups are compact [DS3].

The results are announced in $\S 1$, the proofs of the main theorems are given in $\S 3$, and some preliminary facts and technical tools are given in $\S 2$. We denote by $\mathbb{N}$ the set of natural numbers, and by $\mathbb{Z}_{p}$ the ring, as well as the compact additive group, of $p$-adic integers. The rest of the notation, used in $\S 3$, is introduced in $\S 2$.

Most of the results in this paper were announced in [DS2].

\section{Results}

The theorems which are given, but not proved, in this section will be proved in $\S 3$.

Recall that a topological space $X$ is $\omega$-bounded if each countable subset of $X$ has compact closure in $X$.

1.1. Definition. (i) $\omega-\mathscr{B}$ denotes the class of all $\omega$-bounded groups.

(ii) $\mathscr{C}^{\prime}$ denotes the class of all Abelian groups which can be represented as a union of its countably compact subgroups.

(iii) $\mathscr{C}$ denotes the class of countably compact Abelian groups.

(iv) $\mathscr{P}$ denotes the class of pseudocompact Abelian groups.

One can easily see that $\omega-\mathscr{B} \subseteq \mathscr{C} \subseteq \mathscr{C}^{\prime}$ and $\mathscr{C} \subseteq \mathscr{P}$.

Our first theorem generalizes in the Abelian case the result of Stephenson [S2] mentioned in the introduction. 
1.2. Theorem. Each minimal group $G \in \mathscr{C}^{\prime}$ is perfectly minimal.

1.3. Corollary. Finite products of minimal groups from the class $\mathscr{C}^{\prime}$ are minimal. In particular, if $G \in \mathscr{C}^{\prime}$ is a minimal group, then the group $G^{n}$ is minimal for every natural number $n$.

Proof. The proof is by induction on the size of the product.

1.4. Corollary. If $G$ is a minimal, countably compact Abelian group, then $G^{n}$ is minimal for every natural number $n$.

Since $\omega-\mathscr{B} \subseteq \mathscr{C}^{\prime}$, the next theorem demonstrates that finite products cannot be replaced by infinite ones in the first part of Corollary 1.3.

1.5. Theorem. Let $p$ be a prime number. Then there exists a sequence $\left\{G_{n}: n\right.$ $\in \mathbb{N}\}$ with the following properties: $\mathbb{Z}_{p}^{\omega_{1}}$

(a) each $G_{n}$ is an $\omega$-bounded (hence countably compact), dense subgroup of

(b) for every $n \in \mathbb{N}$ and for each cardinal $\tau$ the group $G_{n}^{\tau}$ is perfectly minimal, and

(c) $\prod\left\{G_{n}: n \in \mathbb{N}\right\}$ is not minimal.

Countably compact, totally minimal Abelian groups are compact [DS3]. Our next corollary shows that "totally minimal" cannot be weakened to "minimal" here.

1.6. Corollary. There exists an $\omega$-bounded (hence countably compact), noncompact, Abelian group $G$ so that each power $G^{\tau}$ of $G$ is perfectly minimal.

Proof. Let $\left\{G_{n}: n \in \mathbb{N}\right\}$ be the sequence from Theorem 1.5. Since $\prod\left\{G_{n}: n \in\right.$ $\mathbb{N}\}$ is not minimal, it cannot be compact. So at least one of the $G_{n}$ 's is not compact. Now take this $G_{n}$ as $G .^{1}$

1.7. Theorem. $\alpha(\omega-\mathscr{B})=\alpha\left(\mathscr{C}^{\prime}\right)=\alpha(\mathscr{C})=\omega$.

1.8. Corollary. $\kappa\left(\mathscr{C}^{\prime}\right) \leq \omega$.

Proof. Combine Theorem 1.7 with Proposition 0.2(iv).

1.9. Corollary. If the countable power $G^{\omega}$ of a countably compact Abelian group $G$ is minimal, then $G^{\sigma}$ is minimal for every cardinal $\sigma$, i.e., $\kappa(\mathscr{C}) \leq$ $\omega$.

In view of Corollaries 1.4 and 1.9 , for the class $\mathscr{C}$ we have either $\kappa(\mathscr{C})=1$ or $\kappa(\mathscr{C})=\omega$, depending on the answer to the following question.

1.10. Question. Is $G^{\omega}$ minimal for a countably compact, minimal Abelian group $G$ ?

Our next theorem demonstrates that the inequality $\kappa(\mathscr{Q}) \leq \alpha(\mathscr{Q})$ in Proposition $0.2(\mathrm{iv})$ cannot be replaced by the equality.

\footnotetext{
${ }^{1}$ By carefully examining the proof of Theorem 1.5 one can easily see that in fact each $G_{n}$ is not compact.
} 
1.11. Theorem. There is a class $\mathscr{Q}$ of topological Abelian groups with $\kappa(\mathscr{Q})<$ $\alpha(\mathscr{Q})$.

Proof. Set $\mathscr{Q}=\left\{G_{n}: n \in \mathbb{N}\right\}$, where $\left\{G_{n}: n \in \mathbb{N}\right\}$ is constructed in Theorem 1.5. It is clear that $\alpha(\mathscr{Q}) \geq \omega$. Conversely, since $\mathscr{Q} \subseteq \mathscr{C}$, it follows that $\alpha(\mathscr{Q}) \leq \alpha(\mathscr{C}) \leq \omega$ (Proposition 0.2 and Theorem 1.7), so $\alpha(\mathscr{Q})=\omega$. On the other hand, $\kappa\left(G_{n}\right)=1$ for every $n \in \mathbb{N}$, so $\kappa(\mathscr{Q})=1$.

Now we turn to the pseudocompact case. Our aim will be to show that in the passage from countably compact groups to pseudocompact ones the situation with $\alpha$ and $\kappa$ changes entirely (compare Corollaries 1.3 and 1.9 with Corollaries $1.13-1.15$ and $1.17-1.19)$.

1.12. Theorem. Let $\tau$ and $\sigma$ be cardinals, $1<\tau<\sigma<\mathfrak{c}$. Assume that $\sigma \geq \omega_{1}$ is a regular cardinal and $2^{\sigma}=\mathfrak{c}$. Then for every prime number $p$ there exists a pseudocompact dense subgroup $G$ of the group $\mathbb{Z}_{p}^{\sigma}$ such that $G^{\lambda}$ is totally minimal for every $\lambda<\tau$, but $G^{\tau}$ is not minimal, so $\kappa(G)=\tau$.

It is worth noting that the additional set-theoretic assumptions in $1.13,1.14$, 1.16 , and 1.17 below are known to be consistent with ZFC, the usual ZermeloFraenkel axioms of set theory.

1.13. Corollary. Suppose that $2^{\omega_{1}}=\mathfrak{c}$. Then for every natural number $n$ there exists a pseudocompact Abelian group $G$ such that $G^{n}$ is totally minimal, but $G^{n+1}$ is not even minimal, so $\kappa(G)=n+1$.

Proof. Put $\tau=n+1$ and $\sigma=\omega_{1}$ in Theorem 1.12.

1.14. Corollary. Suppose that $2^{\omega_{1}}=\mathfrak{c}$. Then there exists a pseudocompact Abelian group $G$ such that $G^{n}$ is totally minimal for every natural number $n$, while $G^{\omega}$ is not minimal, so $\kappa(G)=\omega$.

Proof. Set $\tau=\omega$ and $\sigma=\omega_{1}$ in Theorem 1.12.

Further, the following result holds (roughly speaking, $\kappa(G)$ for a pseudocompact group $G$ can be anything you wish, satisfying, of course, the unique restriction $\kappa(G) \leq \kappa \leq \mathfrak{c}$; see the Introduction).

1.15. Corollary. Let $\alpha \geq 1$ be an ordinal. Then it is consistent with ZFC that there exists a pseudocompact Abelian group $G$ such that $G^{\lambda}$ is totally minimal whenever $\lambda<\omega_{\alpha}$, but $G^{\omega_{\alpha}}$ is not minimal, so $\kappa(G)=\omega_{\alpha}$.

Proof. Using the forcing technique one can construct a model $\mathfrak{M}$ of ZFC in which Martin's Axiom holds and $\mathfrak{c}=\omega_{\alpha+2}[\mathrm{~K}]$. Then $2^{\omega_{\alpha+1}}=\mathfrak{c}$ in $\mathfrak{M}$ and it suffices to put $\tau=\omega_{\alpha}, \sigma=\omega_{\alpha+1}$, and apply Theorem 1.12.

1.16. Theorem. Assume that $2^{\omega_{1}}=\mathfrak{c}$. Let $\sigma$ be a cardinal satisfying $\sigma \leq \mathfrak{c}$ and $2^{\tau}=\mathfrak{c}$ for each infinite cardinal $\tau<\sigma$. Then for every prime $p$ there exists a dense pseudocompact subgroup $G$ of $\mathbb{Z}_{p}^{\omega_{1}}$ such that $G^{\tau}$ is totally minimal for every $\tau<\sigma$, but $G^{\mathfrak{c}}$ is not minimal, so $\kappa(G) \geq \sigma$.

1.17. Corollary. Assume $2^{\omega_{1}}=\mathfrak{c}$. Then $2^{\kappa(\mathscr{P})}>\mathfrak{c}$; in particular, $\kappa(\mathscr{P})>\omega_{1}$.

Proof. Denote by $\sigma$ the smallest cardinal $\lambda$ with $2^{\lambda}>\mathfrak{c}$. It is clear that $\omega_{1}<$ $\sigma \leq \mathfrak{c}$, so $2^{\tau}=\mathfrak{c}$ for every infinite cardinal $\tau<\sigma$. By Theorem 1.16 there is a $G \in \mathscr{P}$ with $\kappa(G) \geq \sigma$. Therefore $\kappa(\mathscr{P}) \geq \kappa(G) \geq \sigma$ and $2^{\kappa(\mathscr{P})} \geq 2^{\sigma}>\mathfrak{c}$. 
Let MA $+\neg \mathrm{CH}$ denote the conjunction of Martin's Axiom together with the negation of the Continuum Hypothesis. This additional set-theoretic assumption is known to be consistent with the usual axioms ZFC of set theory [K].

1.18. Corollary. Suppose $\mathrm{MA}+\neg \mathrm{CH}$. Then there exists a pseudocompact Abelian group $G$ such that $G^{\lambda}$ is totally minimal for each $\lambda<\mathfrak{c}$, but $G^{\mathfrak{c}}$ is not even minimal, so $\kappa(G)=\mathfrak{c}$.

Proof. Under Martin's Axiom, $2^{\tau}=\mathfrak{c}$ for each infinite cardinal $\tau<\mathfrak{c}[\mathrm{K}]$. Set $\sigma=\mathfrak{c}$ in Theorem 1.16, and let $G$ be as in the conclusion of Theorem 1.16. Then $\kappa(G) \geq \mathfrak{c}$. On the other hand, $\kappa(G) \leq \kappa \leq \mathfrak{c}$ by Proposition $0.2(\mathrm{i})$, (iii).

1.19. Corollary. Assume $\mathrm{MA}+\neg \mathrm{CH}$. Then $\alpha(\mathscr{P})=\kappa(\mathscr{P})=\mathfrak{c}$.

Proof. Combine Corollary 1.18 with Proposition 0.2.

1.20. Question. Do all results of 1.12-1.19 hold without any additional settheoretic assumptions beyond ZFC?

The group $G$ in Theorem 1.16 satisfies $w(G)=\omega_{1}$, i.e., it has the minimal possible weight for a pseudocompact noncompact group.

1.21. Question. Is it possible to construct the group $G$ as in Corollary 1.15 with the additional restriction $w(G)=\omega_{1}$ ? ing

By examining Proposition 0.2 and Theorem 1.10 one can suggest the follow-

1.22. Question. Let $\sigma$ and $\tau$ be cardinals satisfying $1 \leq \sigma \leq \tau \leq \mathfrak{c}$. Is there any class $\mathscr{Q}$ of topological Abelian groups with $\alpha(\mathscr{Q})=\tau$ and $\kappa(\mathscr{Q})=\sigma$ ?

\section{Preliminaries}

We denote by $\widehat{G}$ the completion of a topological Abelian group $G$.

Let $G$ be an Abelian group and let $A$ be a subset of $G$. We denote by $\langle A\rangle$ the subgroup of $G$ generated by $A$. If $\langle A\rangle$ is free, the subset $A$ is said to be independent. Each independent subset of $G$ is contained in a maximal one, and all maximal independent subsets of $G$ have the same cardinality, which will be denoted by $r(G)$ (it is called usually the free-rank of $G$ ).

Let $R$ be a commutative ring with unit and let $M$ be a unital $R$-module. An element $x \in M$ is said to be nontorsion if $r \cdot x \neq 0$ whenever $r$ is a nonzero element of $R ; M$ is said to be torsion-free if every nonzero element of $M$ is nontorsion. For a nonempty subset $A$ of $M$ and $B \subseteq M$ we set $[A: B]=\{r \in R: r B \subseteq A\}$.

We denote ordinal numbers by small Greek letters and follow current settheoretic usage by considering any ordinal $\alpha$ as the set of all its predecessors, i.e., the segment $[0, \alpha)$. Thus $\alpha \in \beta$ is understood as $\alpha<\beta$. We also identify the cardinal numbers with the first ordinal having the corresponding cardinality.

For a function $f$ we denote by $\operatorname{dom} f$ and $\operatorname{rng} f$ the domain and the range of $f$, respectively. If $X$ is a set and $\sigma$ is a cardinal, we define

$$
\begin{gathered}
X^{<\sigma}=\{h: h \text { is a function, } \operatorname{dom} h \in \sigma, \operatorname{rng} h \subseteq X\}, \\
{[X]^{<\sigma}=\{F \subseteq X:|F|<\sigma\} .}
\end{gathered}
$$


For $h \in X^{<\sigma}$ and $f \in X^{\sigma}$ we write $h \subseteq f$ if $h(\beta)=f(\beta)$ for each $\beta \in \operatorname{dom} h$.

We list now, in the above notation, the following easy to establish facts.

2.1. Fact. Let $A \neq \varnothing, B$ and $C$ be subsets of $M$, and let $\left\{B_{\gamma}: \gamma<\alpha\right\}$ be a family of subsets of $M$. Then:

(a) $\left[A: \bigcup\left\{B_{\gamma}: \gamma<\alpha\right\}\right]=\bigcap\left\{\left[A: B_{\gamma}\right]: \gamma<\alpha\right\}$; therefore $[A: B]=$ $\bigcap\{[A:\{b\}]: b \in B\}$ if $B \neq \varnothing$ and $[A: \varnothing]=R$ otherwise,

(b) $[A: C]+[B: C] \subseteq[(A+B): C]$ and $[(-A): C]=-[A: C]$; in particular, $[A: C]$ is a subgroup of the additive group of $R$ whenever $A$ is a subgroup of the underlying Abelian group of $M$, and

(c) if $b$ is a nontorsion element of $M$, then $|[A:\{b\}]| \leq|A|$, and so $|[A: B]| \leq|A|$ provided that $B$ contains a nontorsion element of $M$.

The following fact is well known (see [HR]).

2.2. Fact. (a) $|H|=\max \{r(H), \omega\}$ for every torsion-free Abelian group $H$.

(b) For every prime number $p$ the set of invertible elements of the ring $\mathbb{Z}_{p}$ is $\mathbb{Z}_{p} \backslash p \mathbb{Z}_{p}$ and $\left|\mathbb{Z}_{p} \backslash p \mathbb{Z}_{p}\right|=\left|\mathbb{Z}_{p}\right|=r\left(\mathbb{Z}_{p}\right)=\mathfrak{c}$.

Our next fact will be used frequently in $\S 3$. Although it is a particular case of a much stronger result which holds not only for topological groups but also for topological spaces, for the reader's convenience we give its proof here.

2.3. Fact. Let $p$ be a prime number and let $\sigma$ be a regular uncountable cardinal. Then a dense subgroup $G$ of $\mathbb{Z}_{p}^{\sigma}$ is pseudocompact provided that for every $h \in \mathbb{Z}_{p}^{<\sigma}$ there exists a $g \in G$ with $h \subseteq g$.

Proof. According to a theorem of Comfort and Ross [CRs] (see also [C, Theorem 6.4] for an easy proof) $G$ is pseudocompact iff each nonempty $G_{\delta}$-subset of $\widehat{G}=\mathbb{Z}_{p}^{\sigma}$ intersects $G$. So to check this suppose that $x \in U=\bigcap\left\{U_{n}: n \in \mathbb{N}\right\}$, where every $U_{n}$ is open in $\widehat{G}$. Then each $U_{n}-x$ is an open neighborhood of 0 in $\mathbb{Z}_{p}^{\sigma}$, so $\mathbb{Z}_{p}^{\sigma \backslash \gamma_{n}} \subseteq U_{n}-x$ for some $\gamma_{n}<\sigma$ (here for $\gamma<\sigma$ we identify $\mathbb{Z}_{p}^{\sigma \backslash \gamma}$ with the subgroup of those $h \in \mathbb{Z}_{p}^{\sigma}$ which satisfy $h(\beta)=0$ for every $\beta<\gamma$ ). Since $\operatorname{cf}(\sigma)=\sigma>\omega$, it follows that $\gamma=\sup \left\{\gamma_{n}: n \in \mathbb{N}\right\}<\sigma$. On the other hand, $\mathbb{Z}_{p}^{\sigma \backslash \gamma} \subseteq \mathbb{Z}_{p}^{\sigma \backslash \gamma_{n}} \subseteq U_{n}-x$ for any $n \in \mathbb{N}$, thus

$$
x+\mathbb{Z}_{p}^{\sigma \backslash \gamma} \subseteq U .
$$

Consider $h \in \mathbb{Z}_{p}^{<\sigma}$ defined by $\operatorname{dom} h=\gamma$ and $h(\beta)=x(\beta)$ for each $\beta<\gamma$. By hypothesis there exists a $g \in G$ with $h \subseteq g$, and this yields $g-x \in \mathbb{Z}_{p}^{\sigma \backslash \gamma}$. By virtue of (1) we get $g \in U$.

The following minimality criterion will be adopted in our proofs in $\S 3$.

2.4. Minimality criterion. Let $p$ be a prime number, $\sigma$ and $\tau$ arbitrary nonzero cardinals, and $G$ a dense subgroup of $\mathbb{Z}_{p}^{\sigma}$. Then:

(a) $G^{\tau}$ is minimal iff $[G: C] \neq\{0\}$ for each subset $C$ of $\mathbb{Z}_{p}^{\sigma}$ with $|C| \leq \tau$, and

(b) $G^{\tau}$ is totally minimal iff $[G: C] \backslash p \mathbb{Z}_{p} \neq \varnothing$ for each subset $C$ of $\mathbb{Z}_{p}^{\sigma}$ with $|C| \leq \tau$.

Proof. Let us note that $\widehat{G}=\mathbb{Z}_{p}^{\sigma}$, so $\left(G^{\tau}\right)^{\wedge}=(\widehat{G})^{\tau}=\left(\mathbb{Z}_{p}^{\sigma}\right)^{\tau}$. In what follows the $\mathbb{Z}_{p}$-module structure of this group will also be used. We present the proof of the following fact only for the sake of completeness. 
2.5. Fact. Suppose that $\lambda$ is a cardinal, $f \in \mathbb{Z}_{p}^{\lambda}, f \neq 0$, and $N$ is the smallest closed subgroup of $\mathbb{Z}_{p}^{\lambda}$ that contains $f$. Then $N$ is the cyclic $\mathbb{Z}_{p}$-submodule of $\mathbb{Z}_{p}^{\lambda}$ generated by $f$, i.e., $N=\left\{a \cdot f: a \in \mathbb{Z}_{p}\right\}$. Moreover, the mapping $\pi: \mathbb{Z}_{p} \rightarrow N$ defined by $a \rightarrow a \cdot f$ is an isomorphism between topological groups $\mathbb{Z}_{p}$ and $N$, i.e., $N \cong \mathbb{Z}_{p}$.

Proof. The ring $\mathbb{Z}_{p}^{\lambda}$ has the natural structure of a topological $\mathbb{Z}_{p}$-module, so $\pi$ is continuous. Since $f \neq 0$, some of the $\mathbb{Z}_{p}$-coordinates of $f$, say $f_{\alpha}$ for some $\alpha \in \lambda$, differ from 0 . Since $\mathbb{Z}_{p}$ has no zero divisors (see, for example, [DPS, Theorem 3.5.5]), the map $a \rightarrow a \cdot f_{\alpha}$ from $\mathbb{Z}_{p}$ to $\mathbb{Z}_{p}$ is an injection, and hence so is $\pi$. On the other hand, $\pi$ is obviously a group homomorphism from $\mathbb{Z}_{p}$ onto $\pi\left(\mathbb{Z}_{p}\right)$. Thus, $\pi$ is a group isomorphism between $\mathbb{Z}_{p}$ and $\pi\left(\mathbb{Z}_{p}\right)$. Being a one-to-one continuous mapping defined on the compact space $\mathbb{Z}_{p}, \pi$ is a homeomorphism between $\mathbb{Z}_{p}$ and $\pi\left(\mathbb{Z}_{p}\right)$. Being compact, $\pi\left(\mathbb{Z}_{p}\right)$ is a closed subgroup of $\mathbb{Z}_{p}^{\lambda}$. Since $f \in \pi\left(\mathbb{Z}_{p}\right)$ and $N$ is the smallest closed subgroup of $\mathbb{Z}_{p}^{\lambda}$ containing $f$, it follows that $N=\pi\left(\mathbb{Z}_{p}\right)$.

(a) $\Rightarrow$ Assume that $G^{\tau}$ is minimal and fix a subset $C$ of $\mathbb{Z}_{p}^{\sigma}$ with $|C| \leq \tau$. In view of Fact 2.1(a) it is not restrictive to suppose that $|C|=\tau$. Consider an $f \in\left(\mathbb{Z}_{p}^{\sigma}\right)^{\tau}$ such that $\operatorname{rng} f=C$; clearly $f \neq 0$. Let $N$ be the closed subgroup of $\left(\mathbb{Z}_{p}^{\sigma}\right)^{\tau}$ generated by $f$. By the minimality criterion given in [S1], there exists a nonzero $x \in N \cap G^{\tau}$. In view of Fact $2.5, x=a \cdot f$ for some $a \in \mathbb{Z}_{p}$, and of course, $a \neq 0$. Now note that $a \in[G: C]$.

(b) $\Rightarrow$ Assume that $G^{\tau}$ is totally minimal. Because of the total minimality criterion given in [DP] (see also [DPS, Theorem 4.3.3]), with $C, f$, and $N$ as in (a) $\Rightarrow$, we can claim that $N \cap G^{\tau}$ is dense in $N$. Since $N \cong \mathbb{Z}_{p}$ by Fact 2.5 , the subgroup $p N$ is the maximal closed, proper subgroup of $N$, hence the element $x$ can be chosen now with the additional property $x \notin p N$. So the element $a \in \mathbb{Z}_{p}$ from (a) $\Rightarrow$ now satisfies $a \notin p \mathbb{Z}_{p}$. Therefore $[G: C] \backslash p \mathbb{Z}_{p} \neq \varnothing$. The necessity in (a) and (b) is proven.

(a) $\Leftarrow$ Assume that the condition in (a) holds. According to the minimality criterion from [S1], to establish the minimality of $G^{\tau}$ it suffices to prove that

$$
N \cap G^{\tau} \neq\{0\}
$$

for every cyclic $\mathbb{Z}_{p}$-submodule $N$ of $\left(\mathbb{Z}_{p}^{\sigma}\right)^{\tau}$. Let $f$ be a generator of $N$. Then, for $C=\operatorname{rng} f \subseteq \mathbb{Z}_{p}^{\sigma},(2)$ is clearly equivalent to $[G: C] \neq\{0\}$. Since $|C| \leq \tau$, the result follows.

(b) $\Leftarrow$ Assume that the condition in (b) holds. According to the total minimality criterion used in (b) $\Rightarrow$, to establish the total minimality of $G$ instead of (2) the following must be checked in the notation of $(a) \Leftarrow$ :

$$
N \cap G^{\tau} \text { is dense in } N \text {. }
$$

Since $|C| \leq \tau$ and (3) is equivalent to $[G: C] \backslash p \mathbb{Z}_{p} \neq \varnothing$, the condition in the hypothesis can be applied.

\section{Proofs of THE MAIN THEOREMS}

Following [DS1] denote by $\mathscr{B}$ the class of all topological Abelian groups $G$ having the following property: For every prime number $p$, if $x \in G$ is nontorsion and the subgroup topology induced on $\langle x\rangle \simeq \mathbb{Z}$ by the topology 
of $G$ coincides with the $p$-adic topology, then $x$ is contained in a compact subgroup of $G$.

\subsection{Lemma. $\mathscr{C}^{\prime} \subseteq \mathscr{B}$.}

Proof. Suppose that $G \in \mathscr{C}^{\prime}$. Let $p$ be a prime number and let $x \in G$ be such that the subgroup topology on $\langle x\rangle \simeq \mathbb{Z}$ coincides with the $p$-adic topology. Then $\langle x\rangle$ is metrizable, and so is $H$, the smallest closed subgroup of $G$ that contains $x$. Indeed, since $\langle x\rangle$ is first countable and dense in the (regular) space $H$, it follows that $H$ is also first countable [E, Exercise 2.1.C(a)], but for topological groups first countability coincides with metrizability [B, Ka]. Now use the hypothesis of our lemma and choose a countably compact subgroup $N$ of $G$ containing $x$. Then $H_{0}=H \cap N$ is a closed subgroup of $N$, and so it is countably compact. Since countably compact, metrizable spaces are compact [E, Theorem 4.1.17], and $H_{0}$ is metrizable as a subspace of the metrizable space $H$, we conclude that $H_{0}$ is compact. Now note that $x \in H_{0}$.

Proof of Theorem 1.2. Lemma 3.1 implies that $G \in \mathscr{B}$, and the result follows from [DS1, Proposition 1.4].

Proof of Theorem 1.5. Define $K=\mathbb{Z}_{p}^{\omega_{1}}$ and let $F \subseteq \mathbb{Z}_{p}^{\omega_{1}}$ be the $\Sigma$-product, i.e., the set of those $f \in \mathbb{Z}_{p}^{\omega_{1}}$ for which there exists a $\beta \in \omega_{1}$ (depending on $f$ ) such that $f(\alpha)=0$ whenever $\beta<\alpha<\omega_{1}$ [Cs]. One can easily see that $F$ is a subgroup of $\mathbb{Z}_{p}^{\omega_{1}}$, and so $G_{n}=p^{n} K+F$ is also a subgroup of $\mathbb{Z}_{p}^{\omega_{1}}$ for every $n \in \mathbb{N}$. Let us prove that $\left\{G_{n}: n \in \mathbb{N}\right\}$ is the required family. Fix an $n \in \mathbb{N}$ and consider $G_{n}$.

(i) Since $F$ is $\omega$-bounded (see, for example, [C, Theorem 6.10]) and $K$ is compact, the product $K \times F$ is $\omega$-bounded. The mapping $\varphi: K \times F \rightarrow K$, defined by $\varphi(x, y)=p^{n} \cdot x+y$ for $(x, y) \in K \times F$, is continuous, so $G_{n}=$ $\varphi(K \times F)$ is $\omega$-bounded as a continuous image of an $\omega$-bounded space.

(ii) Note that for each $m \in \mathbb{N}$

$$
p^{m} \widehat{G}_{n} \subseteq G_{n} \text { iff } n \leq m,
$$

which yields that $G_{n}^{\tau}$ is perfectly minimal for each cardinal $\tau$ [D] (see also [DS1, Corollary 3.6] or [DPS, Corollary 6.1.17]).

(iii) By Lemma 3.1, $\left\{G_{n}: n \in \mathbb{N}\right\} \subseteq \omega-\mathscr{B} \subseteq \mathscr{C}^{\prime} \subseteq \mathscr{B}$. Since (4) holds for each $n \in \mathbb{N}$, we can apply [DS1, (a) $\Leftrightarrow(\mathrm{c})$ of Theorem 2.4 and Lemma 2.1.d)] to conclude that $\prod\left\{G_{n}: n \in \mathbb{N}\right\}$ is not minimal (see also [DPS, Theorem 6.2.7]).

Proof of Theorem 1.7. From Lemma 3.1 it follows that $\omega-\mathscr{B} \subseteq \mathscr{C} \subseteq \mathscr{C}^{\prime} \subseteq$ $\mathscr{B}$, so $\alpha(\omega-\mathscr{B}) \leq \alpha(\mathscr{C}) \leq \alpha\left(\mathscr{C}^{\prime}\right) \leq \alpha(\mathscr{B})$ by Proposition 0.2(ii). Theorem 1.5 implies $\alpha(\omega-\mathscr{B}) \geq \omega$, and the inequality $\alpha(\mathscr{B}) \leq \omega$ follows from [DS1, Theorem 4.1] (see also [DPS, Theorem 6.2.7]).

In the proof of the remaining theorems two algebraic lemmas will be needed. No recourse to topology is made there.

3.2. Lemma. Suppose that $R$ is a ring with unit, $M$ is a torsion-free R-module, $G$ and $H$ are subgroups of the additive group $(M,+)$ of the $R$-module $M, X$ is an independent subset of $(M,+)$, and $r(H)<|X|$. Define

$$
S=\{r \in R:[(G+r \cdot H): X] \backslash[G: X] \neq \varnothing\} .
$$

Then $|S| \leq \max (\omega,|X|,|G|)$. 
Proof. Set $\tau=\max (\omega,|X|,|G|)$ and $\tilde{X}=\langle X\rangle \backslash\{0\}$. For every $h \in H$ define

$$
T_{h}=[(A \cdot X+G):\{h\}], \quad \text { where } A=\bigcup\{[G:\{x\}]: x \in \tilde{X}\},
$$

and set $T=\bigcup\left\{T_{h}: h \in H \backslash\{0\}\right\}$. Since $M$ is torsion-free and $0 \notin \tilde{X}$, from Fact 2.1(c) it follows that $|A| \leq \tau$, and therefore

$$
|A \cdot X+G| \leq \max (\omega,|X|,|G|)=\tau .
$$

Applying Fact 2.1(c) once more, we conclude that $\left|T_{h}\right| \leq \tau$ for every $h \in$ $H \backslash\{0\}$. Since $|H| \leq \max (\omega, r(H))$ (Fact $2.2(\mathrm{a}))$ and $r(H)<|X| \leq \tau$, one obtains that $|H| \leq \tau$ and hence $|T| \leq \tau$. So to prove our lemma it suffices to show that $S \subseteq T$. Fix an $r \in S$. From the definition of $S$ it follows that there exists an $a \in R$ such that $a \in[(G+r \cdot H): X] \backslash[G: X]$.

Since $a \in[(G+r \cdot H): X]$, for every $x \in X$ we can choose a $g_{x} \in G$ and an $h_{x} \in H$ such that

$$
a \cdot x=g_{x}+r \cdot h_{x} .
$$

Since $r(H)<|X|$, one can find distinct elements $x_{0}, \ldots, x_{k} \in X$ and integers $n_{0}, \ldots, n_{k}$ such that $\sum_{i=0}^{k} n_{i} h_{x_{i}}=0$ and $n_{j} \neq 0$ for some $j \leq k$. Then

$$
\begin{aligned}
a \cdot\left(\sum_{i=0}^{k} n_{i} x_{i}\right) & =\sum_{i=0}^{k} n_{i} \cdot a \cdot x_{i}=\sum_{i=0}^{k} n_{i} \cdot\left(g_{x_{i}}+r \cdot h_{x_{i}}\right) \\
& =\sum_{i=0}^{k} n_{i} g_{x_{i}}+r \cdot\left(\sum_{i=0}^{k} n_{i} h_{x_{i}}\right)=\sum_{i=0}^{k} n_{i} g_{x_{i}} \in G .
\end{aligned}
$$

Since $X$ is an independent set, $\sum_{i=0}^{k} n_{i} x_{i} \neq 0$; hence $\sum_{i=0}^{k} n_{i} x_{i} \in \tilde{X}$ and $a \in A$.

On the other hand, from (5) and $a \notin[G: X]$ it follows that $h_{x^{*}} \neq 0$ for some $x^{*} \in X$. Then

$$
r \cdot h_{x^{*}}=a \cdot x^{*}-g_{x^{*}} \in A \cdot X+G,
$$

and therefore $r \in T_{h_{x^{*}}}$. Now $h_{x^{*}} \neq 0$ yields $r \in T$. So $S \subseteq T$.

3.3. Lemma. Suppose $M$ is a torsion-free $R$-module over a ring $R$ with unit, $G, H$, and $Y$ are subsets of $M$, and $G \cap Y=\varnothing$. Let

$$
P=\{r \in R:(G+r \cdot H) \cap Y \neq \varnothing\} .
$$

Then $|P| \leq \max (\omega,|G|,|H|,|Y|)$.

Proof. For each $h \in H$ define $Q_{h}=[(Y-G):\{h\}]$ and let $Q=\bigcup\left\{Q_{h}: h \in\right.$ $H \backslash\{0\}\}$. From Fact $2.1(\mathrm{c})$ it follows that $\left|Q_{h}\right| \leq \max (\omega,|G|,|Y|)$ for each $h \in H \backslash\{0\}$, so $|Q| \leq \max (\omega,|G|,|H|,|Y|)$. Now to finish the proof it suffices to show that $P \subseteq Q$. Fix an $r \in P$. Then there are $g \in G, h \in H$, and $y \in Y$ with $y=g+r \cdot h$. Therefore, $r \in Q_{h}$. Note that $h \neq 0$, since otherwise we would have $g=y$ contradicting $G \cap Y=\varnothing$.

A nonlimit ordinal $\beta+n$, where $\beta$ is a limit ordinal and $n \in \mathbb{N}$, is said to be odd (even) if $n$ is odd (even). 
We begin first with the proof of Theorem 1.16 which is technically easier than that of Theorem 1.12.

Proof of Theorem 1.16. The symbol 1 denotes the element of $\mathbb{Z}_{p}^{\omega_{1}}$ having the unit $1 \in \mathbb{Z}_{p}$ as all its coordinates. If $\gamma \in \omega_{1}$, then $\pi_{\gamma}$ denotes the canonical projection of $\mathbb{Z}_{p}^{\omega_{1}}$ onto $\mathbb{Z}_{p}^{\omega_{1} \backslash \gamma}$.

Using our cardinal assumption we can enumerate the set $\left[\mathbb{Z}_{p}^{\omega_{1}}\right]^{<\sigma}$ by odd nonlimit ordinals less than $\mathfrak{c}$, and the set $\mathbb{Z}_{p}^{<\omega_{1}}$ by even nonlimit ordinals less than $\mathfrak{c}$. Let

$$
\left[\mathbb{Z}_{p}^{\omega_{1}}\right]^{<\sigma}=\left\{F_{\alpha}: \alpha<\mathfrak{c}, \alpha \text { is an odd nonlimit ordinal }\right\}
$$

and

$$
\mathbb{Z}_{p}^{<\omega_{1}}=\left\{g_{\alpha}: \alpha<\mathfrak{c}, \alpha \text { is an even nonlimit ordinal }\right\}
$$

be such enumerations. Set $F_{-1}=\varnothing$ for convenience.

For every $\alpha<\mathfrak{c}$ we will construct by transfinite recursion a subgroup $G_{\alpha}$ of $\mathbb{Z}_{p}^{\omega_{1}}$ satisfying the following conditions:

$\left(1_{\alpha}\right)$ if $\beta<\alpha$, then $G_{\beta} \subseteq G_{\alpha}$,

$\left(2_{\alpha}\right) \quad\left|G_{\alpha}\right|<\mathfrak{c}$,

$\left(3_{\alpha}\right)$ if $\alpha$ is an odd nonlimit ordinal, then

$$
\left(\mathbb{Z}_{p} \backslash p \mathbb{Z}_{p}\right) \cap\left[G_{\alpha}: F_{\alpha}\right] \neq \varnothing,
$$

$\left(4_{\alpha}\right)$ if $\alpha$ is an even nonlimit ordinal, then $g_{\alpha} \subseteq g$ for some $g \in G_{\alpha}$, and $\left(\mathbf{5}_{\alpha}\right) \quad \pi_{\gamma}\left(G_{\alpha}\right) \cap \pi_{\gamma}(\langle\mathbf{1}\rangle)=\{0\}$ whenever $\gamma \in \omega_{1}$.

The above conditions are trivially fulfilled if we put $G_{-1}=\{0\}$.

Inductive step. Let $\alpha$ be an ordinal, $0 \leq \alpha<\mathfrak{c}$, and suppose that for $\beta<\alpha$ all subgroups $G_{\beta}$ satisfying $\left(1_{\beta}\right)-\left(5_{\beta}\right)$ have already been defined. We consider three cases.

Case 1: $\alpha$ is a limit ordinal. In this case it suffices to define $G_{\alpha}=\bigcup\left\{G_{\beta}: \beta \in\right.$ $\alpha\}$.

Case 2: $\alpha=\beta+1, \alpha$ is odd. Define $H_{\alpha}=\left\langle F_{\alpha}\right\rangle$ and

$$
P_{\gamma}=\left\{a \in \mathbb{Z}_{p}: \pi_{\gamma}\left(G_{\beta}+a \cdot H_{\alpha}\right) \cap \pi_{\gamma}(\langle\mathbf{1}\rangle) \neq\{0\}\right\}
$$

for each $\gamma \in \omega_{1}$. Then $\left|H_{\alpha}\right| \leq\left|F_{\alpha}\right|+\omega<\mathfrak{c}$. Fix a $\gamma \in \omega_{1}$. From $\left(5_{\beta}\right)$ it follows that $R=\mathbb{Z}_{p}, M=\mathbb{Z}_{p}^{\omega_{1} \backslash \gamma}, G=\pi_{\gamma}\left(G_{\beta}\right), H=\pi_{\gamma}\left(H_{\alpha}\right)$, and $Y=\pi_{\gamma}(\langle\mathbf{1}\rangle) \backslash\{0\}$ satisfy the assumptions of Lemma 3.3. This yields $\left|P_{\gamma}\right|<\mathfrak{c}$, taking into account $\left(2_{\beta}\right)$ and the equality $\pi_{\gamma}\left(G_{\beta}+a \cdot H_{\alpha}\right)=G+a \cdot H$. So for $P=\bigcup\left\{P_{\gamma}: \gamma \in \omega_{1}\right\}$ we obtain $|P|<\mathfrak{c}$, and we can choose an element $a \in \mathbb{Z}_{p} \backslash\left(p \mathbb{Z}_{p} \cup P\right.$ ) (see Fact 2.2(b)). Now, by our choice, conditions $\left(1_{\alpha}\right)-\left(5_{\alpha}\right)$ would be satisfied for $G_{\alpha}=G_{\beta}+a \cdot H_{\alpha}$.

Case 3: $\alpha=\beta+1$ is an even nonlimit ordinal. Applying the argument from Case 2 to $G_{\beta}$ and $H_{\alpha}=Y=\langle\mathbf{1}\rangle$, we get an element $a \in \mathbb{Z}_{p} \backslash p \mathbb{Z}_{p}$ such that, for $G_{\alpha}^{\prime}=G_{\beta}+a \cdot\langle\mathbf{1}\rangle$,

$$
\pi_{\gamma}\left(G_{\alpha}^{\prime}\right) \cap \pi_{\gamma}(\langle\mathbf{1}\rangle)=\{0\} \quad \text { whenever } \gamma \in \omega_{1} .
$$

Consider the element $f \in \mathbb{Z}_{p}^{\omega_{1}}$ defined by

$$
f(\gamma)= \begin{cases}a^{-1} \cdot g_{\alpha}(\gamma) & \text { if } \gamma \in \operatorname{dom} g_{\alpha} \\ 1 & \text { otherwise }\end{cases}
$$


Let $G_{\alpha}=G_{\beta}+a \cdot\langle f\rangle$. Then properties $\left(1_{\alpha}\right)-\left(3_{\alpha}\right)$ are trivially satisfied, and $\left(4_{\alpha}\right)$ follows from $g_{\alpha} \subseteq a \cdot f \in G_{\alpha}$. It remains to check $\left(5_{\alpha}\right)$.

For $\gamma \in \omega_{1}$ fixed, there is a $\gamma^{*} \in \omega_{1}$ such that $\gamma^{*}>\gamma$ and $\gamma^{*} \in \omega_{1} \backslash$ dom $g_{\alpha}$. If $\pi_{\gamma}\left(G_{\alpha}\right) \cap \pi_{\gamma}(\langle\mathbf{1}\rangle) \neq\{0\}$, then also

$$
\pi_{\gamma^{*}}\left(G_{\alpha}\right) \cap \pi_{\gamma^{*}}(\langle\mathbf{1}\rangle) \neq\{0\} .
$$

By the definition of $f$ and the choice of $\gamma^{*}, \pi_{\gamma^{*}}(f)=\pi_{\gamma^{*}}(\mathbf{1})$, so

$$
\begin{aligned}
\pi_{\gamma^{*}}\left(G_{\alpha}^{\prime}\right) & =\pi_{\gamma^{*}}\left(G_{\beta}+a \cdot\langle\mathbf{1}\rangle\right)=\pi_{\gamma^{*}}\left(G_{\beta}\right)+a \cdot \pi_{\gamma^{*}}(\langle\mathbf{1}\rangle) \\
& =\pi_{\gamma^{*}}\left(G_{\beta}\right)+a \cdot \pi_{\gamma^{*}}(\langle f\rangle)=\pi_{\gamma^{*}}\left(G_{\beta}+a \cdot\langle f\rangle\right)=\pi_{\gamma^{*}}\left(G_{\alpha}\right) .
\end{aligned}
$$

Now (9) implies

$$
\pi_{\gamma^{*}}\left(G_{\alpha}^{\prime}\right) \cap \pi_{\gamma^{*}}(\langle\mathbf{1}\rangle)=\pi_{\gamma^{*}}\left(G_{\alpha}\right) \cap \pi_{\gamma^{*}}(\langle\mathbf{1}\rangle) \neq\{0\},
$$

which contradicts (8). The inductive step is finished.

Now define $G=\bigcup\left\{G_{\alpha}: \alpha<\mathfrak{c}\right\}$. Since $\left(5_{\alpha}\right)$ with $\gamma=0$ holds for all $\alpha<\mathfrak{c}$, we conclude that

$$
G \cap\langle\mathbf{1}\rangle=\{0\} .
$$

If $G^{\mathfrak{c}}$ were minimal, then $G$ must be perfectly minimal [S3] (see also [DS1, Corollary 3.6] or [DPS, Corollary 6.1.17]); on the other hand, (10) implies that $G$ is not perfectly minimal [S3] (see also [DPS, Theorem 6.1.8], where item d) fails). Thus $G^{\mathfrak{c}}$ is not minimal. From $\left(3_{\alpha}\right)$ for all $\alpha<\mathfrak{c},(6)$, and Minimality Criterion 2.4(b) it follows that $G^{\lambda}$ is totally minimal for each $\lambda<\sigma$. Furthermore, properties $\left(4_{\alpha}\right)$ for $\alpha<\mathfrak{c},(7)$, and Fact 2.3 yield the pseudocompactness of $G$.

Proof of Theorem 1.12. The symbol 1 denotes the point of $\mathbb{Z}_{p}^{\sigma}$ having the unit $1 \in \mathbb{Z}_{p}$ as all its coordinates. Fix an independent set $\left\{c_{\delta}: \delta \in \tau\right\}$ in $\mathbb{Z}_{p}$ (this is possible because of $\tau<\mathfrak{c}$ and Fact 2.2(b)). Now set $\mathbf{c}_{\delta}=c_{\delta} \cdot \mathbf{1}$ for $\delta \in \tau$ and $\mathbf{C}=\left\{\mathbf{c}_{\delta}: \delta \in \tau\right\}$. An immediate consequence of our choice is the following

3.4. Claim. For each $\gamma \in \sigma, \pi_{\gamma}(\mathbf{C})$ is an independent subset of $\mathbb{Z}_{p}^{\sigma \backslash \gamma}$ and $\left|\pi_{\gamma}(\mathbf{C})\right|=\tau<\mathfrak{c}$.

According to our cardinal assumption, we can enumerate the set $\left[\mathbb{Z}_{p}^{\sigma}\right]^{<\tau}$ by odd nonlimit ordinals less than $\mathfrak{c}$, and the set $\mathbb{Z}_{p}^{<\sigma}$ by even nonlimit ordinals less than $c$. Let

$$
\left[\mathbb{Z}_{p}^{\sigma}\right]^{<\tau}=\left\{F_{\alpha}: \alpha<\mathfrak{c}, \alpha \text { is an odd nonlimit ordinal }\right\}
$$

and

$$
\mathbb{Z}_{p}^{<\sigma}=\left\{g_{\alpha}: \alpha<\mathfrak{c}, \alpha \text { is an even nonlimit ordinal }\right\}
$$

be such enumerations. Set $F_{-1}=\varnothing$ for convenience.

Now for every $\alpha<\mathfrak{c}$ we will construct by transfinite recursion a subgroup $G_{\alpha}$ of $\mathbb{Z}_{p}^{\sigma}$ and a set $Y_{\alpha} \subseteq \mathbb{Z}_{p}^{\sigma}$ satisfying the following conditions:

$\left(1_{\alpha}\right)$ if $\beta<\alpha$, then $G_{\beta} \subseteq G_{\alpha}$ and $Y_{\beta} \subseteq Y_{\alpha}$,

$\left(2_{\alpha}\right) \quad\left|G_{\alpha}\right|<\mathfrak{c}$ and $\left|Y_{\alpha}\right|<\mathfrak{c}$,

(3) $\pi_{\gamma}\left(G_{\alpha}\right) \cap \pi_{\gamma}\left(Y_{\alpha}\right)=\varnothing$ for each $\gamma \in \sigma$,

$\left(4_{\alpha}\right)$ if $\alpha$ is an odd nonlimit ordinal, then

$$
\left(\mathbb{Z}_{p} \backslash p \mathbb{Z}_{p}\right) \cap\left[G_{\alpha}: F_{\alpha}\right] \neq \varnothing,
$$


$\left(5_{\alpha}\right)$ if $\alpha$ is an even nonlimit ordinal, then $g_{\alpha} \subseteq g$ for some $g \in G_{\alpha}$, and

$\left(6_{\alpha}\right)$ if $\gamma \in \sigma, b \in \mathbb{Z}_{p} \backslash\{0\}, \delta \in \tau$, and $b \cdot \pi_{\gamma}\left(\mathbf{c}_{\delta}\right) \in \pi_{\gamma}\left(G_{\alpha}\right)$, then $b \cdot \mathbf{c}_{\mu} \in Y_{\alpha}$ for some $\mu \in \tau$.

Before starting the recursion let us mention the following

3.5. Claim. If $G_{\alpha}$ and $Y_{\alpha}$ satisfy $\left(3_{\alpha}\right)$ and $\left(6_{\alpha}\right)$, then $\left[\pi_{\gamma}\left(G_{\alpha}\right): \pi_{\gamma}(\mathbf{C})\right]=\{0\}$ for each $\gamma \in \sigma$.

Proof. Suppose the contrary, and let $b \in\left[\pi_{\gamma}\left(G_{\alpha}\right): \pi_{\gamma}(\mathbf{C})\right] \backslash\{0\}$ for some $\gamma \in \sigma$. Since $0 \in \tau, b \cdot \pi_{\gamma}\left(\mathbf{c}_{0}\right) \in \pi_{\gamma}\left(G_{\alpha}\right)$. Then, according to $\left(6_{\alpha}\right)$, there exists a $\mu \in \tau$ such that $b \cdot \mathbf{c}_{\mu} \in Y_{\alpha}$, and so $b \cdot \pi_{\gamma}\left(\mathbf{c}_{\mu}\right) \in \pi_{\gamma}\left(Y_{\alpha}\right)$. Now $\left(3_{\alpha}\right)$ yields that $b \cdot \pi_{\gamma}\left(\mathbf{c}_{\mu}\right) \notin \pi_{\gamma}\left(G_{\alpha}\right)$; thus, $b \notin\left[\pi_{\gamma}\left(G_{\alpha}\right): \pi_{\gamma}(\mathbf{C})\right]$, a contradiction.

Basis of induction. Set $G_{-1}=\{0\}$ and $Y_{-1}=\varnothing$ for convenience. Then $\left(1_{-1}\right)-\left(6_{-1}\right)$ are satisfied trivially.

Inductive step. Let $\alpha$ be an ordinal, $0 \leq \alpha<\mathfrak{c}$, and suppose that for all $\beta<\alpha$ subgroups $G_{\beta} \subseteq \mathbb{Z}_{p}^{\sigma}$ and sets $Y_{\beta} \subseteq \mathbb{Z}_{p}^{\sigma}$ satisfying $\left(1_{\beta}\right)-\left(6_{\beta}\right)$ have already been defined. We consider three different cases.

Case 1: $\alpha$ is a limit ordinal. Define $G_{\alpha}=\bigcup\left\{G_{\beta}: \beta \in \alpha\right\}$ and $Y_{\alpha}=\bigcup\left\{Y_{\beta}: \beta \in\right.$ $\alpha\}$. Then conditions $\left(1_{\alpha}\right),\left(2_{\alpha}\right),\left(4_{\alpha}\right)$, and $\left(5_{\alpha}\right)$ are trivially satisfied. For the verification of $\left(3_{\alpha}\right)$ and $\left(6_{\alpha}\right)$ the following lemma can be applied.

3.6. Lemma. Suppose that $\alpha \leq \mathfrak{c}$ and, for every $\beta<\alpha, G_{\beta}$ and $Y_{\beta}$ satisfy $\left(1_{\beta}\right)-\left(6_{\beta}\right)$. Then $G_{\alpha}=\bigcup\left\{G_{\beta}: \beta \in \alpha\right\}$ and $Y_{\alpha}=\bigcup\left\{Y_{\beta}: \beta \in \alpha\right\}$ satisfy $\left(3_{\alpha}\right)$ and $\left(6_{\alpha}\right)$.

Proof. Assume $f \in \pi_{\gamma}\left(G_{\alpha}\right) \cap \pi_{\gamma}\left(Y_{\alpha}\right)$ for some $\gamma \in \sigma$. Then there are $\beta_{1}, \beta_{2} \in \alpha$ with $f \in \pi_{\gamma}\left(G_{\beta_{1}}\right)$ and $f \in \pi_{\gamma}\left(Y_{\beta_{2}}\right)$. So for $\beta=\max \left(\beta_{1}, \beta_{2}\right)$ we would have

$$
f \in \pi_{\gamma}\left(G_{\beta_{1}}\right) \cap \pi_{\gamma}\left(Y_{\beta_{2}}\right) \subseteq \pi_{\gamma}\left(G_{\beta}\right) \cap \pi_{\gamma}\left(Y_{\beta}\right) \neq \varnothing,
$$

contradicting $\left(3_{\beta}\right)$. This proves $\left(3_{\alpha}\right)$.

As for $\left(6_{\alpha}\right)$, suppose that $\gamma \in \sigma, b \in \mathbb{Z}_{p} \backslash\{0\}, \delta \in \tau$, and $b \cdot \pi_{\gamma}\left(\mathbf{c}_{\delta}\right) \in \pi_{\gamma}\left(G_{\alpha}\right)$. Since $G_{\alpha}=\bigcup\left\{G_{\beta}: \beta \in \alpha\right\}$, it follows that $b \cdot \pi_{\gamma}\left(\mathbf{c}_{\delta}\right) \in \pi_{\gamma}\left(G_{\beta}\right)$ for some $\beta \in \alpha$. Now $\left(6_{\beta}\right)$ implies $b \cdot c_{\mu} \in Y_{\beta} \subseteq Y_{\alpha}$ for some $\mu \in \tau$.

Case 2: $\alpha=\beta+1, \alpha$ is odd. Define $H_{\alpha}=\left\langle F_{\alpha}\right\rangle$, and

$$
\begin{aligned}
P_{\gamma} & =\left\{a \in \mathbb{Z}_{p}: \pi_{\gamma}\left(G_{\beta}+a \cdot H_{\alpha}\right) \cap \pi_{\gamma}\left(Y_{\beta}\right) \neq \varnothing\right\}, \\
Q_{\gamma} & =\left\{a \in \mathbb{Z}_{p}:\left[\pi_{\gamma}\left(G_{\beta}+a \cdot H_{\alpha}\right): \pi_{\gamma}(\mathbf{C})\right] \neq\{0\}\right\}
\end{aligned}
$$

for every $\gamma \in \sigma$. Then $\left|H_{\alpha}\right| \leq \max \left(\omega,\left|F_{\alpha}\right|\right)<\mathfrak{c}$.

3.7. Claim. $\left|P_{\gamma}\right|<\mathrm{c}$ and $\left|Q_{\gamma}\right|<\mathrm{c}$ for each $\gamma \in \sigma$.

Proof. Fix a $\gamma \in \sigma$. From $\left(3_{\beta}\right)$ it follows that $R=\mathbb{Z}_{p}, M=\mathbb{Z}_{p}^{\sigma \backslash \gamma}, G=$ $\pi_{\gamma}\left(G_{\beta}\right), H=\pi_{\gamma}\left(H_{\alpha}\right)$, and $Y=\pi_{\gamma}\left(Y_{\beta}\right)$ satisfy all the assumptions of Lemma 3.3 , which yields

$$
\left|P_{\gamma}\right| \leq \max \left(\omega,\left|G_{\beta}\right|,\left|H_{\alpha}\right|,\left|Y_{\beta}\right|\right)<\mathfrak{c},
$$

because $G+a \cdot H=\pi_{\gamma}\left(G_{\beta}+a \cdot H_{\alpha}\right)$. On the other hand,

$$
r\left(\pi_{\gamma}\left(H_{\alpha}\right)\right) \leq r\left(H_{\alpha}\right) \leq\left|F_{\alpha}\right|<\tau=\left|\pi_{\gamma}(\mathbf{C})\right|,
$$


and $\pi_{\gamma}(\mathbf{C})$ is an independent subset of $\mathbb{Z}_{p}^{\sigma \backslash \gamma}$ (see Claim 3.4). So $R, M, G, H$ as above and $X=\pi_{\gamma}(\mathbf{C})$ satisfy the assumptions of Lemma 3.2. This gives

$$
\left|S_{\gamma}\right| \leq \max \left(\omega,\left|\pi_{\gamma}(\mathbf{C})\right|,\left|\pi_{\gamma}\left(G_{\beta}\right)\right|\right) \leq \max \left(\omega,|\mathbf{C}|,\left|G_{\beta}\right|\right)<\mathfrak{c},
$$

where

$$
S_{\gamma}=\left\{a \in \mathbb{Z}_{p}:\left[\pi_{\gamma}\left(G_{\beta}+a \cdot H_{\alpha}\right): \pi_{\gamma}(\mathbf{C})\right] \backslash\left[\pi_{\gamma}\left(G_{\beta}\right): \pi_{\gamma}(\mathbf{C})\right] \neq \varnothing\right\} .
$$

Since $\left(3_{\beta}\right)$ and $\left(6_{\beta}\right)$ hold, $\left[\pi_{\gamma}\left(G_{\beta}\right): \pi_{\gamma}(\mathbf{C})\right]=\{0\}$ (Claim 3.5); thus, $S_{\gamma}=Q_{\gamma}$ and $\left|Q_{\gamma}\right|=\left|S_{\gamma}\right|<\mathfrak{c}$.

Let $\Phi=\bigcup\left\{P_{\gamma} \cup Q_{\gamma}: \gamma \in \sigma\right\}$. Then $\sigma<\mathfrak{c}$ and the above claim imply $|\Phi|<\mathfrak{c}$, and since $\left|\mathbb{Z}_{p} \backslash p \mathbb{Z}_{p}\right|=\mathfrak{c}$ (Fact 2.2(b)), we can choose an $a \in \mathbb{Z}_{p} \backslash\left(p \mathbb{Z}_{p} \cup \Phi\right)$. Define $G_{\alpha}=G_{\beta}+a \cdot H_{\alpha}$. By our choice, $\left|G_{\alpha}\right|<\mathfrak{c}, G_{\beta} \subseteq G_{\alpha}$, and $a \in\left[G_{\alpha}: F_{\alpha}\right]$, so $\left(1_{\alpha}\right),\left(2_{\alpha}\right)$ for $G_{\alpha}$, and $\left(4_{\alpha}\right)$ hold. We have also

3.8. Claim. $\pi_{\gamma}\left(G_{\alpha}\right) \cap \pi_{\gamma}\left(Y_{\beta}\right)=\varnothing$ and $\left[\pi_{\gamma}\left(G_{\alpha}\right): \pi_{\gamma}(\mathbf{C})\right]=\{0\}$ for each $\gamma \in$ $\sigma$.

To define $Y_{\alpha}$ we need the following

3.9. Lemma. Beyond the hypothesis of Theorem 1.12 suppose additionally that $G \subseteq \mathbb{Z}_{p}^{\sigma}$ and $\left[\pi_{\gamma}(G): \pi_{\gamma}(\mathbf{C})\right]=\{0\}$ for every $\gamma \in \sigma$. Then there exists an $X \subseteq \mathbb{Z}_{p}^{\sigma}$ such that

(i) $|X| \leq \max (\sigma,|G|)$,

(ii) $\pi_{\gamma}(G) \cap \pi_{\gamma}(X)=\varnothing$ whenever $\gamma \in \sigma$, and

(iii) if $b \in \mathbb{Z}_{p} \backslash\{0\}, \gamma \in \sigma, \delta \in \tau$, and $b \cdot \pi_{\gamma}\left(\mathbf{c}_{\delta}\right) \in \pi_{\gamma}(G)$, then $b \cdot \mathbf{c}_{\mu} \in X$ for some $\mu \in \tau$.

Proof. Fix a $\gamma \in \sigma$ and a $\delta \in \tau$. Since $\pi_{\gamma}\left(\mathbf{c}_{\delta}\right) \neq 0$, for $B_{\gamma, \delta}=\left[\pi_{\gamma}(G):\left\{\pi_{\gamma}\left(\mathbf{c}_{\delta}\right)\right\}\right]$ we would have $\left|B_{\gamma, \delta}\right| \leq\left|\pi_{\gamma}(G)\right| \leq|G|$ (Fact 2.1(c)). Hence if one defines $B=\bigcup\left\{B_{\gamma, \delta}: \gamma \in \sigma, \delta \in \tau\right\}$, then $|B| \leq \max (\sigma,|G|)$ (recall that $\left.\tau<\sigma\right)$. Since $\left[\pi_{\gamma}(G): \pi_{\gamma}(\mathbf{C})\right]=\{0\}$ whenever $\gamma \in \sigma$, for $b \in B \backslash\{0\}$ and $\gamma \in \sigma$ we can find a $\delta(b, \gamma) \in \tau$ with $b \cdot \pi_{\gamma}\left(\mathbf{c}_{\delta(b, \gamma)}\right) \notin \pi_{\gamma}(G)$. Since $\tau<\sigma$ and $\sigma$ is a regular cardinal, there are $\Sigma(b) \subseteq \sigma$ and $\delta_{b} \in \tau$ such that $\Sigma(b)$ is cofinal in $\sigma$ and $\delta_{b}=\delta(b, \gamma)$ for any $\gamma \in \bar{\Sigma}(b)$. First of all note that

$$
b \cdot \pi_{\gamma}\left(\mathbf{c}_{\delta_{b}}\right) \notin \pi_{\gamma}(G) \text { for each } \gamma \in \sigma .
$$

Indeed, $\Sigma(b)$ is cofinal in $\sigma$, so for any $\gamma \in \sigma$ we can find a $\xi \in \Sigma(b)$ with $\gamma<\xi$. Then $b \cdot \pi_{\xi}\left(\mathbf{c}_{\delta_{b}}\right) \notin \pi_{\xi}(G)$, and consequently $b \cdot \pi_{\gamma}\left(\mathbf{c}_{\delta_{b}}\right) \notin \pi_{\gamma}(G)$.

Now for $X=\left\{b \cdot \mathbf{c}_{\delta_{b}}: b \in B \backslash\{0\}\right\}$ we have $|X| \leq|B| \leq \max (\sigma,|G|)$, and (13) implies (ii). To check (iii) observe that if $b, \gamma$, and $\delta$ satisfy the assumption of (iii), then $b \in B_{\gamma, \delta}$ and so $b \in B \backslash\{0\}$; now it remains to apply the definition of $X$.

To finish the inductive step in Case 2, using Claim 3.8, apply Lemma 3.9 to $G=G_{\alpha}$ and define $Y_{\alpha}=Y_{\beta} \cup X$, where $X$ is constructed in Lemma 3.9. Then $\left(1_{\alpha}\right)$ for $Y_{\alpha}$ is trivial; condition $\left(2_{\alpha}\right)$ for $Y_{\alpha}$ follows from $\sigma<\mathfrak{c},\left(2_{\beta}\right)$, and Lemma 3.9(i); $\left(3_{\alpha}\right)$ holds because $\pi_{\gamma}\left(G_{\alpha}\right) \cap \pi_{\gamma}(X)=\varnothing$ (Lemma 3.9(ii)) and $\pi_{\gamma}\left(G_{\alpha}\right) \cap \pi_{\gamma}\left(Y_{\beta}\right)=\varnothing$ (Claim 3.8); and $\left(6_{\alpha}\right)$ follows from Lemma 3.9(iii).

Case 3: $\alpha=\beta+1$ is an even nonlimit ordinal. Since $\tau>1$, we can apply to $G_{\beta}, Y_{\beta}$, and $H_{\alpha}=\langle\mathbf{1}\rangle$ the argument from the above case to obtain a subgroup 
$G_{\alpha}^{\prime}=G_{\beta}+a \cdot\langle\mathbf{1}\rangle$ (with $a \in \mathbb{Z}_{p}$ ) and a subset $Y_{\alpha} \supseteq Y_{\beta}$ of $\mathbb{Z}_{p}^{\sigma}$ such that $\left|Y_{\alpha}\right|<\mathfrak{c}$,

$$
\pi_{\gamma}\left(G_{\alpha}^{\prime}\right) \cap \pi_{\gamma}\left(Y_{\alpha}\right)=\varnothing \quad \text { for each } \gamma \in \sigma \text {, }
$$

and

$$
\begin{aligned}
& \text { if } \gamma \in \sigma, b \in \mathbb{Z}_{p} \backslash\{0\}, \delta \in \tau, \text { and } b \cdot \pi_{\gamma}\left(\mathbf{c}_{\delta}\right) \in \\
& \pi_{\gamma}\left(G_{\alpha}^{\prime}\right), \text { then } b \cdot \mathbf{c}_{\mu} \in Y_{\alpha} \text { for some } \mu \in \tau .
\end{aligned}
$$

Consider the element $f \in \mathbb{Z}_{p}^{\sigma}$ defined by

$$
f(\gamma)= \begin{cases}a^{-1} \cdot g_{\alpha}(\gamma) & \text { if } \gamma \in \operatorname{dom} g_{\alpha}, \\ 1 & \text { otherwise. }\end{cases}
$$

Let $G_{\alpha}=G_{\beta}+a \cdot\langle f\rangle$. Conditions $\left(1_{\alpha}\right),\left(2_{\alpha}\right)$, and $\left(4_{\alpha}\right)$ are satisfied in a trivial way, and $\left(5_{\alpha}\right)$ follows from $g_{\alpha} \subseteq a \cdot f \in G_{\alpha}$. Let us verify $\left(3_{\alpha}\right)$. For $\gamma \in \sigma$ fixed, choose a $\gamma^{*} \in \sigma \backslash$ dom $g_{\alpha}$ with $\gamma^{*}>\gamma$. Note that

$$
\begin{aligned}
\pi_{\gamma^{*}}\left(G_{\alpha}\right) & =\pi_{\gamma^{*}}\left(G_{\beta}+a \cdot\langle f\rangle\right)=\pi_{\gamma^{*}}\left(G_{\beta}\right)+a \cdot \pi_{\gamma^{*}}(\langle f\rangle) \\
& =\pi_{\gamma^{*}}\left(G_{\beta}\right)+a \cdot \pi_{\gamma^{*}}(\langle\mathbf{1}\rangle)=\pi_{\gamma^{*}}\left(G_{\beta}+a \cdot\langle\mathbf{1}\rangle\right)=\pi_{\gamma^{*}}\left(G_{\alpha}^{\prime}\right) .
\end{aligned}
$$

Now (14) implies

$$
\pi_{\gamma^{*}}\left(G_{\alpha}\right) \cap \pi_{\gamma^{*}}\left(Y_{\alpha}\right)=\pi_{\gamma^{*}}\left(G_{\alpha}^{\prime}\right) \cap \pi_{\gamma^{*}}\left(Y_{\alpha}\right)=\varnothing,
$$

which yields $\pi_{\gamma}\left(G_{\alpha}\right) \cap \pi_{\gamma}\left(Y_{\alpha}\right)=\varnothing$ because $\gamma<\gamma^{*}$.

As for $\left(6_{\alpha}\right)$, assume that $\gamma \in \sigma, b \in \mathbb{Z}_{p} \backslash\{0\}, \delta \in \tau$, and $b \cdot \pi_{\gamma}\left(\mathbf{c}_{\delta}\right) \in \pi_{\gamma}\left(G_{\alpha}\right)$. Choose a $\gamma^{*} \in \sigma \backslash$ dom $g_{\alpha}$ with $\gamma^{*}>\gamma$. Then $b \cdot \pi_{\gamma^{*}}\left(\mathbf{c}_{\delta}\right) \in \pi_{\gamma^{*}}\left(G_{\alpha}\right)=\pi_{\gamma^{*}}\left(G_{\alpha}^{\prime}\right)$, and (15) implies the existence of $\mu \in \tau$ for which $b \cdot \mathbf{c}_{\mu} \in Y_{\alpha}$.

Finally, define $G=G_{\mathfrak{c}}=\bigcup\left\{G_{\alpha}: \alpha<\mathfrak{c}\right\}$ and $Y=Y_{\mathfrak{c}}=\bigcup\left\{Y_{\alpha}: \alpha<\mathfrak{c}\right\}$. Lemma 3.6 implies that conditions $\left(3_{\mathfrak{c}}\right)$ and $\left(6_{\mathfrak{c}}\right)$ hold for $G_{\mathfrak{c}}$ and $Y_{\mathfrak{c}}$, hence $[G: \mathbf{C}]=\left[G_{\mathfrak{c}}: \mathbf{C}\right]=\{0\}$ according to Claim 3.5. Thus $G^{\tau}$ is not minimal by Minimality Criterion 2.4. On the other hand, (11), condition $\left(4_{\alpha}\right)$ for all $\alpha<\mathfrak{c}$, and the same criterion yield total minimality of $G^{\lambda}$ for every $\lambda<\tau$. Pseudocompactness of $G$ follows from (12), condition $\left(5_{\alpha}\right)$ for all $\alpha<\mathfrak{c}$, and Fact 2.3.

\section{ACKNOWLEDGMENT}

The authors would like to thank the referee for helpful comments on the first version of this paper.

\section{REFERENCES}

[B] G. Birkhoff, A note on topological groups, Compositio Math. 3 (1936), 427-430.

[C] W. W. Comfort, Topological groups, Handbook of Set-Theoretic Topology (K. Kunen and J. E. Vaughan, eds.), North-Holland, Amsterdam-New York-Oxford, 1984, pp. 1143-1263.

[CG] W. W. Comfort and D. L. Grant, Cardinal invariants, pseudocompactness and minimality: some recent advances in the topological theory of topological groups, Topology Proc. 6 (1981), 227-265.

[CRb] W. W. Comfort and L. C. Robertson, Cardinality constraints for pseudocompact and for totally dense subgroups of compact topological groups, Pacific J. Math. 119 (1985), 265285.

[CRs] W. W. Comfort and K. A. Ross, Pseudocompactness and uniform continuity in topological groups, Pacific J. Math. 16 (1966), 483-496. 
[CS] W. W. Comfort and T. Soundararajan, Pseudocompact group topologies and totally dense subgroups, Pacific J. Math. 100 (1982), 61-84.

[Cs] H. H. Corson, Normality in subsets of product spaces, Amer. J. Math. 81 (1959), 785-796.

[D] D. Dikranjan, Sur la minimalité des produits de groupes topologiques abéliens, C. R. Acad. Sci. Paris Sér. I 299 (1984), 303-306.

[DP] D. Dikranjan and Iv. Prodanov, Totally minimal topological groups, Annuaire Univ. Sofia Fac. Math. Méc. 69 (1974/75), 5-11.

[DPS] D. Dikranjan, Iv. Prodanov, and L. Stoyanov, Topological groups: characters, dualities and minimal group topologies, Monographs and Textbooks Pure Appl. Math., vol. 130, Marcel Dekker, New York-Basel, 1989.

[DS1] D. Dikranjan and D. Shakhmatov, Products of minimal abelian groups, Math. Z. 204 (1990), 583-603.

[DS2] _ Critical power of minimality of topological groups close to being compact, C. R. Acad. Bulgare Sci. 43 (10) (1990), 13-15.

[DS3] _ Compact-like totally dense subgroups of compact groups, Proc. Amer. Math. Soc. 114 (1992), 1119-1129.

[Do] D. Doitchinov, Produits de groupes topologiques minimaux, Bull. Sci. Math. (2) 97 (1972), 59-64.

[E] R. Engelking, General topology, PWN, Warsaw, 1977.

[F] M. Fréchet, Sur quelques points du calcul fonctionel, Rend. Circ. Mat. Palermo 22 (1906), $1-74$.

[G] I. I. Guran, On minimal topological groups, Topology and Set Theory, Udmurt State University, Izhevsk, 1982, pp. 64-71. (Russian)

[H] E. Hewitt, Rings of real-valued continuous functions. I, Trans. Amer. Math. Soc. 64 (1948), 45-99.

[HR] E. Hewitt and K. Ross, Abstract harmonic analysis, Vol. 1, Springer-Verlag, Berlin-Heidelberg-New York, 1963.

[Ka] S. Kakutani, Über die Metrisation der topologischen Gruppen, Proc. Imperial Acad. Tokyo 12 (1936), 82-84.

[Ku] K. Kunen, Set theory. An introduction to independence proofs, Studies in Logic and Foundations of Math., vol. 102, North-Holland, Amsterdam-New York-Oxford, 1980.

[PS1] Iv. Prodanov and L. Stoyanov, Every minimal abelian group is precompact, C. R. Acad. Bulgare Sci. 37 (1) (1984), 23-26.

[PS2] _ Minimal group topologies, Topology and its Applications (Proc. Eger Topol. Conf., Eger (Hungary), 1983), Colloq. Math. Soc. János Bolyai, vol. 41, North-Holland, Amsterdam-New York-Oxford, 1985, pp. 493-508.

[S1] R. M. Stephenson, Jr., Minimal topological groups, Math. Ann. 192 (1971), 193-195.

[S2] _ Some unsolved problems concerning P-minimal and P-closed spaces, Proc. Charlotte Topological Conf., Academic Press, New York, 1974, pp. 249-257.

[S3] L. Stoyanov, On products of minimal and totally minimal groups, Proc. 11 th Spring Conf. of the Union of Bulgarian Math., Sunny Beach, 1982, pp. 287-294.

[S4] _. Weak periodicity and minimality of topological groups, Annuaire Univ. Sofia Fac. Math. Méc. 73 (1978/79), 155-167.

Institute of Mathematics, Bulgarian Academy of Sciences, 1090 Sofia, Bulgaria

Current address: Department of Mathematics and Informatics, University of Udine, via Zanon 6, 33100 Udine, Italy

E-mail address: dikranja@udmi5400.cineca.it

Department of Mechanics and Mathematics, Moscow State University, 119899 Moscow, Russia

Current address: Department of Mathematics, University of Toronto, Toronto, Ontario, Canada M5S 1A1

E-mail address: dshakhmatov@miavx1.bitnet 